\title{
Enhancing Resonance Imaging Sensitivity and Upconversion Emission of Gd-based Nanoscale Coordination Polymers by Combination With Au Nanoparticles
}

\section{Chen Xiao}

Research Center for Ultrafine Powder Materials, College of Chemistry and Chemical Engineering, Jiangxi Normal University, Nanchang 330022, China.

\section{Tuck Yun Change}

Breast Disease Center, The First Affiliated Hospital, Sun Yat-Sen University, Guangzhou 510080, China

\section{Yin Li}

Research Center for Ultrafine Powder Materials, College of Chemistry and Chemical Engineering, Jiangxi Normal University, Nanchang 330022, China.

\section{Hualan Xu}

Research Center for Ultrafine Powder Materials, College of Chemistry and Chemical Engineering, Jiangxi Normal University, Nanchang 330022, China.

\section{Shengliang Zhong ( $\nabla$ slzhong@jxnu.edu.cn )}

Research Center for Ultrafine Powder Materials, College of Chemistry and Chemical Engineering, Jiangxi Normal University, Nanchang 330022, China. https://orcid.org/0000-0001-5900-9884

\section{Research Article}

Keywords: Nanoscale coordination polymers. highly tailorable structure, bioimaging, biosensing and clinical diagnosis

Posted Date: April 19th, 2021

DOl: https://doi.org/10.21203/rs.3.rs-420997/v1

License: (c) (i) This work is licensed under a Creative Commons Attribution 4.0 International License. Read Full License 


\section{Abstract}

Nanoscale coordination polymers (NCPs) are attracting increasing research interest due to their highly tailorable structure, feasible functionality and promising application in various fields, especially in biomedical applications, they are excellent contrast agents for bioimaging, biosensing and clinical diagnosis. Currently, to overcome the low relaxation rate and unitary information of magnetic resonance imaging, the design of multifunction contrast agent is critical and challenging. Herein, a new $\mathrm{Au}$ nanoparticles/Gd-based coordination polymer (Au@GdCP) composite nanoparticles have been fabricated by a facile hydrothermal methodology. The Au@GdCP exhibit excellent magnetic resonance, bright upconversion luminescence (UC) and good biocompatibility. More importantly, with the introduction of Au nanoparticles, the magnetization and longitudinal relaxation rate $(\mathrm{r} 1)$ of the nanocomposite is enhanced significantly, with 131.6 -fold from $5.19 \mathrm{emu} / \mathrm{g}$ to $683.52 \mathrm{emu} / \mathrm{g}$ and 11.5 fold from $0.248 \mathrm{mM}^{-1} \mathrm{~s}^{-1}$ to $2.853 \mathrm{mM}^{-1} \mathrm{~S}^{-1}$ enhancement, respectively, as well as better UC property over GdCPs. This work may offer a new way to develop multifunctional NCPs with enhanced performance in image-guided diagnosis and therapy.

\section{Introduction}

Magnetic resonance imaging (MRI) is a kind of non-radioactive imaging technology [1, 2]. It is an extremely important clinical diagnosis model for diseased tissues, including solid tumours, tissues, organs, and blood vessels. MRI has several advantages over other diagnosis models because it does not require ionizing radiation, can provide high spatial resolution and high-contrast three-dimensional images [3-5]. It functions as a contrast imaging by changing the longitudinal and transverse relaxation times of the body's water environment [6]. At present, there are many nanocarriers that can be used for MRI, such as noble metal nanoparticles, silica, and rare earth nanomaterials [7].

In particular, $\mathrm{Gd} 3$ + has a large magnetic moment due to its seven unpaired electrons, its compounds are widely used as magnetic contrast agents for clinical diagnosis [8]. For example, Gd-DTPA is one of the most used MRI agents. However, it is limited by the low relaxation rate and nephrotoxicity [9]. To address these problems, various efforts that focus on enhancing the relaxation rate of contrast agents and reducing their toxicity have been developed [10]. Among the techniques used, surface modification is an effective method to enhance MRI signals. For example, carbon quantum dots with good optical properties are used to modify Gd-based materials to improve relaxation rates [11]. Contrast agent materials coated with $\mathrm{SiO}_{2}$ have been reported to improve the relaxivity information [12]. Other strategies have also emerged. For example, a radionuclide-Cu-64 labelled doxorubicin (DOX)-loaded polydopamine (PDA) gadolinium-metallofullerene core-satellite nano-theragnostic agent is developed for multimodal imagingguided combination cancer therapy [13]. It was found that the compound showed good biocompatibility, strong near infrared (NIR) light absorption, and high relaxation rate. Hai et al. designed a Gd-based probe


weighted MR contrast [14]. Another strategy for enhancing MRI signals is to control the internal structure 
of the nanomaterials, such as the formation of core-shell structures and heterojunction structures. Adah Almutairi et al. reported a modulation of the interfacial layer thickness to enhance the MRI signal [15]. Recently, Ye et al. exploited an MRI material through liposome surface modified (cRGD@MLP-Gd) exhibiting $T_{1}$ and $T_{2}$ weighted contrast enhancement in vivo [16]. Compared to the single modal imaging, multimodal imaging probe is significant not only for the great improved detection sensitivity, but also for decreasing the toxicity risk and other side effect [15]. Thereby providing accurate diagnosis and precise therapy.

The most developed nanostructured multimodal imaging probe is the combination of MRI and optical imaging [17], which can overcome the limitations of each modal to generate more comprehensive imaging information with high spatial resolution, infinite tissue penetration and high sensitivity. Gd-based nanomaterials have been proved to be an ideal candidate for multifunctional materials, which combine with upconversion luminesces and MRI [18]. Lanthanide-doped CP upconversion nanoparticles demonstrate an anti-Stokes behavioural process, which converts continuous low energy (such as NIR light or IR light) into high energy light (visible light) by continuous multiphoton absorption or energy transfer processes $[19,20]$, providing a broad range of applications, from electrochemistry to biomedicine $[21,22]$. One of the major attractions for biomedical application of lanthanide-doped upconversion nanoparticles (UCNPs) is that the anti-Stokes process can eliminate background autofluorescence and light scattering effects of the biological material [23]. The other conspicuous attraction is the irradiation light can achieve high tissue penetration depth and have low toxicity to the tissue [24]. Nevertheless, the overall upconversion efficiency is relatively low, and their practical application for biomedical are still limited [25]. As a result, it is desirable to develop simple and versatile synthetic routes for UCNPs with enhanced performance.

In recent years, nanoscale CPs have aroused extensive research interest in the fields of chemistry, physics, medicine, nanotechnology, and materials [26-29]. Particularly, in biomedical, they are excellent contrast agents for bioimaging benefiting from the highly tunable composition and structures, they are intrinsically biodegradable and easy to combine multiple modalities [30]. Furthermore, by combining with suitable materials, the properties of CPs can be improved, with enhanced functionality/stability, ease of preparation, and selectivity of operation [31]. It is expected that CP composites may find extensive applications in near future [32-35]. However, to date, few reports have appeared on the preparation and application of GdCP composites, and rare work studying GdCPs with enhanced MRI effects. Based on the localized surface plasmon resonance (LSPR) effect of Au nanoparticles (AuNPs) and the limitations of its low relaxation rate and potential biotoxicity of Gd-based nano-compounds [36].

In this work, we present the synthesis and characterization of a new MRI/upconverstion luminescence multimodal contrast agent, the as-obtained GdCP nanospheres have good uniformity and monodispersity, the effect of Au NPs used for enhancing upconversion luminesces, relaxation information, and T1-weight MRI and biocompatibility of Au@GdCP has also been explored. Au@GdCP may offer great potential for future biomedical applications. 


\section{Results And Discussion}

\section{Synthesis of Au@GdCP nanocomposites}

The preparation method of Au@GdCP composite nanoparticles contains the following processes. Firstly, AuNPs were synthesized by reduction of chloroauric acid $\left(\mathrm{HAuCl}_{4}, 1 \mathrm{~mL}, 0.025 \mathrm{~mol} / \mathrm{L}\right)$ under sodium citrate solution $(3 \mathrm{~mL}, 0.035 \mathrm{~mol} / \mathrm{L})$ and hexadecyl trimethyl ammonium bromide (CTAB, $20 \mathrm{~mL}, 0.125$ $\mathrm{mol} / \mathrm{L}$ ) was used as stabilizer, the gold nanoparticles (AuNPs) with an average diameter of about $20 \mathrm{~nm}$ was diluted with DI water to $5 \mathrm{~mL}$. Afterwards, $0.2 \mathrm{mmol}$ rare earth ions solution which contains $\mathrm{Gd}\left(\mathrm{NO}_{3}\right)_{3}, \mathrm{Yb}\left(\mathrm{NO}_{3}\right)_{3}$ and $\mathrm{Er}\left(\mathrm{NO}_{3}\right)_{3}$ (molar ratio of $\mathrm{Gd}: \mathrm{Yb}: \mathrm{Er}=0.16: 0.03: 0.01$ ) were add into allantoin solution $(0.3 \mathrm{mmol})$ stirring for $15 \mathrm{~min}$ at room temperature. Subsequently, the above solution was mixed with together and transferred into a Teflon lined stainless-steel autoclave with capacity of $50 \mathrm{~mL}$, heated at $160{ }^{\circ} \mathrm{C}$ for $15 \mathrm{~h}$. As the autoclave was cooled to room temperature naturally. Finally, the product was isolated by centrifugation and washed with deionized water and absolute ethanol for three times, and dried in vacuum at $60{ }^{\circ} \mathrm{C}$ for $12 \mathrm{~h}$. The products marked as Au@GdCP. Meanwhile, the GdCP prepared with same method without Au NP introduced.

\section{Characterization}

The morphologies and sizes of the samples were obtained through scanning electron microscope (SEM, HITACHI S-3400N, 30 kV) and transmission electron microscope (TEM, Hitachi JEM2100, 200 kV). XRD pattern were performed on a Rigaku X-ray diffractometer with Cu Ka radiation $(\lambda=154178 \AA$ ) with the range of $5-90^{\circ}$, FT-IR data was obtained with Nicolet 6700 FT-IR spectrometer using KBr pellet between $4000 \mathrm{~cm}-1$ and $500 \mathrm{~cm}-1$ at room temperature. Photoluminescence (PL) emission spectra were recorded with an FLS 980 (Edinburgh Instruments, England). Elemental analysis was carried out on an EA3000 elemental analyzer. Magnetic properties were measured using a SQUID magnetometer equipment. The relaxation information was performed on a 0.5T NMI20-Analyst NMR system (Niumag Corporation, Shanghai, China).

\section{Characterization of GdCP and Au@GdCP}

The morphologies and sizes of the as-obtained GdCPs were initially characterized by scanning electron microscopic (SEM). As can be seen in Figure. 1(a), the products were discrete and spherical, with a mean diameter of $\sim 179 \mathrm{~nm}$ and relative narrow size distribution, as determined by dynamic light scattering (DLS, insert of Figure. 1a). TEM images under different magnifications (Figure. 1b and c) further verify that GdCP is uniformly round and monodispersed. Interestingly, after combination with Au nanoparticles, A decrease in the particle size was obtained, with an average size of $150 \mathrm{~nm}$ (Figure. 1d) for Au@GdCP, which is helpful for the biomedical applications. The reduction in diameter probably due to the Au particles acting as the nuclei, leading to a decrease in the size of the final product, which is always observed in the growth of the crystal. The TEM image in Figure. 1e shows the internal structure of Au@GdCP. It can be clearly observed that each nanosphere encapsulating several Au nanoparticles. 
Magnification of the TEM image in Figure.1f demonstrate that the Au@GdCP nanosphere has an outer layer with thickness of approximately $50 \mathrm{~nm}$. In addition, the energy dispersive spectroscopy (EDS) mapping analysis of a single nanosphere are illustrated in Figure. $1 \mathrm{~g}$, the $\mathrm{C}, \mathrm{O}, \mathrm{N}, \mathrm{Gd}, \mathrm{Yb}$, and Er elements coexist and are distributed homogeneously throughout the particle. However, the Au element is aggregated and located in the internal part of the Gd-based coordination polymers. All this evidence verifies that AuNPs have successful embedded within GdCP.

To assess the structure of the compounds, powder X-ray diffraction (XRD) patterns of GdCP spheres and Au@GdCP nanocomposites are presented in Figure. 2. No obvious diffraction peak is observed for GdCP, indicating that GdCP is amorphous and not crystalline. While the Au@GdCP exhibits four distinct peaks at $2 \theta=38.37^{\circ}, 44.56^{\circ}, 64.75^{\circ}, 77.72^{\circ}$, which can be assigned to the (111), (200), (220) and (311) planes of the standard data for hexagonal phase of Au (JCPDS 04-0784). The HRTEM image (Figure.1f) shows lattice fringe with the interplanar spacing of $0.252 \mathrm{~nm}$, corresponding to the (200) planes of $\mathrm{Au}$. The result was further proved the presence of AuNPs in the product and the formation of Au@GdCP.

\section{Magnetic properties}

The magnetic properties of GdCP and Au@GdCP nanocomposites were measured by SQUID magnetometer. Figure. 3 shows the magnetization of the magnetic field $(\mathrm{M}-\mathrm{H})$ curves of samples at 300 K. The magnetization of GdCP and Au@GdCP are $5.19 \mathrm{emu} / \mathrm{g}$ and $683.52 \mathrm{emu} / \mathrm{g}$ at $300 \mathrm{k}$, respectively. With the introduction of AuNPs, the magnetization was enhanced remarkably. No hysteresis loop was observed. The magnetization curve of the samples displayed typical paramagnetic properties. These results show that even after introduction of AuNPs, the particle keep well paramagnetic properties.

\section{Luminescence properties of Au@GdCP}

The upconversion emission spectrum of Au@GdCP under 980 nm laser excitation is shown in Figure. 4. Simultaneously, GdCP was used as the control under the same conditions. In this system, $\mathrm{Yb}^{3+}$ acts as a sensitizer and transfers the absorbed excitation energy to the activator ions of $\mathrm{Er}^{3+}$. First, $\mathrm{Yb}^{3+}$ is excited from the state level $\left({ }^{7} \mathrm{~F}_{7 / 2}\right)$ to the exciting level $\left({ }^{5} \mathrm{~F}_{5} / 2\right)$ under the continuous $980 \mathrm{~nm}$ laser excitation. Subsequently, $\mathrm{Er}^{3+}$ ions are excited to the intermediate state $\left({ }^{4} \mathrm{I}_{11} / 2\right)$ and then transfer energy to the excited state $\left({ }^{4} \mathrm{~F}_{7 / 2}\right)$, which resulting the energy transfer of $\mathrm{Er}^{3+}$ ions from ${ }^{4} \mathrm{~F}_{7 / 2}$ levels to the levels of ${ }^{2} \mathrm{I}_{11 / 2},{ }^{4} \mathrm{~S}_{3 / 2}$, and ${ }^{4} \mathrm{H}_{9 / 2}$ with nonradioactive process, respectively [37-39]. The emission spectra exhibited three bands, attributed to green emission ( $525 \mathrm{~nm}$ and $545 \mathrm{~nm})$ and red emission $(655 \mathrm{~nm})$. Compared with GdCP, an obvious enhanced emission intensity of $38.4 \%$ was obtained after Au ions modification, coupled with a slight blue shift for $4 \mathrm{~nm}$, mainly attributed to the LSPR effect of the AuNPs [38]. LSPR strengthens the local crystal field, resulting in the enhancement of excitation field intensity [41]. Meanwhile, coupling between the broad LSPR peaks of AuNPs and the upconversion luminescence enhances the energy transfer effect, improving the decay rate of luminescent centres and the efficiency of the emission intensity $[42,43]$. Therefore, AuNPs play a role in enhancing the fluorescent intensity in 


\section{Relaxation information and $\mathrm{T}_{1}$-weighted MRI maps}

Gd-based composites have good kinetic and thermodynamic stabilities. They can accelerate the $T_{1}$ relaxation of water protons, and improve the brightness of MRI signals. Approximately $40 \%$ of MRI scans employ $\mathrm{Gd}^{3+}$ chelates for contrast agent in clinical [44]. However, free $\mathrm{Gd}^{3+}$ ions are known to have serious toxic effect due to the replacement of $\mathrm{Ca}^{2+}$ ions in the body, further improvement is required. Thanks to the biocompatibility and tuneable morphology, AuNPS have been frequently used in clinical applications. In recent years, they have been exploited to create very promising tools for multimodal imaging and MRI-guided therapies [45]. To estimate the performance of GdCP and Au@GdCP as MRI contrast agent, the relaxation rate and $\mathrm{T}_{1}$-weighted MRI were investigated in vitro at the low field of $0.5 T$. As shown in Figure. 5(a, c), with the increase of Gd concentration, the longitudinal relaxation time and transverse relaxation time of GdCP showed a good linear relationship. Based on the slop, the longitudinal relativity $\left(r_{1}\right)$ and transverse relativity $\left(r_{2}\right)$ were calculated to be $0.248 \mathrm{mM}^{-1} \mathrm{~S}^{-1}$ and $1.292 \mathrm{mM}^{-1} \mathrm{~S}^{-1}$, respectively. In comparsion with GdCP, Au@GdCP exhibited increased values with $2.853 \mathrm{mM}^{-1} \mathrm{~S}^{-1}, 13.797$ $\mathrm{mM}^{-1} \mathrm{~S}^{-1}$ for $\mathrm{r}_{1}, \mathrm{r}_{2}$, respectively. Calculated to be 11.5 fold and 10.8 fold enhancements. The appealing improvement of the Au@GdCP is mainly due to the confined tumbling of $\mathrm{Gd}^{3+}$ in a biomacromolecule, which result a longer rotational correlation time. Besides, compared with previous reported modified UCNPs materials (such as the UCNPs@mSiO 2 -PEG nanocomposite with a $r_{1}$ of $0.6361 \mathrm{mM}^{-1} \mathrm{~s}^{-1}$ ), our results exhibited stronger signals. [46]. The excellent longitudinal and transverse relaxation information with relativel high $r_{2} / r_{1}$ ratio $\left(r_{2} / r_{1}=4.83>3\right)$ of Au@GdCP is expected to achieve $T_{1}-T_{2}$ dual mode MRI.

The T1-weighted MRI of Au@GdCP and GdCP nanocomposites were also obtained (Fig. 3b, d), in which T1-weighted MRI signals got brighter and brighter with the increasing concentration of $\mathrm{Gd}^{3+}$. Compared with the $T_{1}$-weight MRI signal of both nanocomposites, the contrast effect of Au@GdCP is much better than GdCP, demonstrating that the existence of AuNPs greatly enhances the MRI signal intensity and the contrast of the MRI signal.

It is well known that reducing the inversion rate of the contrast agent in the aqueous proton solution can reduce the average spin-nuclear spin and the dipole-dipole interaction between the contrast agent, and it can prolong the inversion time, which can improve the relaxation efficiency of the contrast agent [25]. With the introduction of AuNPs, the LSPR of the AuNPs may strengthen the external magnetic field, which results in intermolecular dipole-dipole interactions, increasing the contrast relaxation rate and the $T_{1}$ weighted MRI signal [47-48]. Moreover, the strong light scattering of AuNPs translates the absorbed light to the plasmon wavelength, resulting in enhancement of $\mathrm{T}_{1}$-weighted MRI signals [49].

\section{Biocompatibility of the Au@GdCPs}

As is well known, good biocompatibility is an important prerequisite and crucial factor for biological applications. In this work. the in vitro biocompatibility of Au@GdCP was evaluated through the 3-(4,5Loading [MathJax]/jax/output/CommonHTML/jax.js 
dimethylthiazol-2-yl)-2,5- diphenyltetrazolium bromide (MTT) assay with incubated HeLa cells. Meanwhile, GdCP was tested as a control under the same conditions. Figure. 6 shows that with the increasing concentration of Au@GdCP and GdCP, the relative cell viability of HeLa gradually decreased. When the concentration of Au@GdCP and GdCP reached $1.0 \mathrm{mg} / \mathrm{mL}$, the relative cell viability is $80 \%$ and $82.2 \%$, respectively. The MTT results indicate that Au@GdCP exhibit low toxicity and good biocompatibility.

\section{Conclusions}

In summary, Au@GdCP nanocomposites were fabricated through a facile hydrothermal method. With the increasing concentration of GdCP and Au@GdCP, the longitudinal relaxation time and lateral relaxation time exhibited an excellent linear relationship, and $\mathrm{T}_{1}$-weight MRI displayed brighter and brighter. When AuNPs were introduced into GdCP, based on the LSPR of AuNPs, the longitudinal relaxation rate of Au@GdCP was much higher than that of GdCP. Meanwhile, Au@GdCP displayed good upconversion luminesces and excellent biocompatibility. These properties demonstrated that Au@GdCPs can be effectively applied to MRI/ upconverstion luminescence multimodal contrast agent and are promising for biomedical applications.

\section{Declarations}

Acknowledge

We acknowledge from Jiangxi Provincial Department of Science and Technology (Nos. 20192BBEL50017 and 20202ACBL203002) and the National Natural Science Foundation of China (No. 22061020).

Compliance with ethical standards

Conflicts of interest. There are no conflicts to declare.

\section{References}

1. Wahsner, Eric.M.Gale, A.Rodríguez-Rodríguez and P. Caravan, Chem. Rev. 119, 957-1057 (2019).

2. T. Moonen, P. C. van Zijl, J. A. Frank, D. Le Bihan and E. D. Becker, Science. 250, 53-61 (1990).

3. Liu, W. Bu and J. Shi, Chem. Rev. 117, 6160-6224 (2017).

4. R. Smith and S. S. Gambhir, Chem. Rev. 117, 901-986 (2017).

5. Hu, S. Mignani, J. Majoral, M. W. Shen and X. Y. Shi, Chem. Soc. Rev. 47, 1874-1900 (2018).

6. Carril, J. Mater. Chem. B 5, 4332-4347 (2017).

7. Pan, L. e. Zhang, L. Zeng, W. Ren, X. Xiao, J. Zhang, L. Zhang, A. Li, G. Lu and A. Wu, Nanoscale 8, 878-888 (2016). 
8. Viswanathan, Z. Kovacs, K. N. Green, S. J. Ratnakar and A. D. Sherry, Chem. Rev. 110, 2960-3018 (2010).

9. Shen, J. Song, Z. Zhou, B. C. Yung, M. A. Aronova, Y. Li, Y. Dai, W. Fan, Y. Liu, Z. Li, H. Ruan, R. D. Leapman, L. Lin, G. Niu, X. Chen and A. Wu, Adv. Mater. 30, 1803163. (2018)

10. J. L. Villaraza, A. Bumb and M. W. Brechbiel, Chem. Rev. 110, 2921-2959 (2010).

11. Du, L. Zhang, L. Zhang, M. Zhang, A. Gong, Y. Tan, J. Miao, Y. Gong, M. Sun, H. Ju, C. Wu and S. Zou, Biomaterials 121, 109-120 (2017).

12. Cho, W. Park, and D. H. Kim, ACS Appl. Mater. Interfaces 9, 101-111 (2017).

13. Wang, J. Lin, Z. Wang, Z. Zhou, R. Bai, N. Lu, Y. Liu, X. Fu, O. Jacobson, W. Fan, J. Qu, S. Chen, T. Wang, P. Huang and X. Chen, Adv. Mater. 29, 1701013 (2017).

14. Hai, Y. Ni, D. Saimi, H. Yang, H. Tong, K. Zhong and G. Liang, Nano lett. 19, 2428-2433 (2019).

15. He, N. J. J. Johnson, V. A. N. Huu, E. Cory, Y. Huang, R. L. Sah, J. V. Jokerst and A. Almutairi, Nano Lett. 17, 4873-4880 (2017).

16. Ye, Y. Liu, Y. Lu, Y. Ji, L. Mei, M. Yang, X. Gong, Q. Gu, D. Li, F. Yang and C.J. Li, J. Mater. Chem. B 8, 447-453 (2020).

17. P. Shi, X. Sun, S. H. Zheng, J. L. Li, X. Y. Fu and H. W. Zhang, Biomaterials 152, 15-23 (2018).

18. Wen, K. Li, H. Cai, Q. Chen, M. Shen, Y. Huang, C. Peng, W. Hou, M. Zhu, G. Zhang and X. Shi, Biomaterials 34, 1570-1580 (2013).

19. Zhu, Y. Ding, A. Wang, X. Sun, X.-C. Wu and J.-J. Zhu, J. Mater. Chem. B 3, 458-464 (2015).

20. S. Quah, W. Chen, M. K. Schreyer, H. Yang, M. W. Wong, W. Ji and J. J. Vittal, Nat. Commun. 6, 7954 (2015).

21. Li, J. Tang, L. He, Y. Liu, Y. Liu, C. Chen, Z. Tang, Adv. Mater. 27, 4075-4080 (2015).

22. Medishetty, J. K. Zareba, D. Mayer, M. Samoc and R. A. Fischer, Chem. Soc. Rev. 46, 4976-5004 (2017).

23. Sun, R. Wei, J. Feng and H. Zhang, Coordin. Chem. Rev. 364, 10-32 (2018).

24. Ju, X. Chen, F. Ai, D. Peng, X. Lin, W. Kong, P. Shi, G. Zhu and F. Wang, J. Mater. Chem. B 3, 3548-3555 (2015).

25. J.Garfield, N. J. Borys, S M. Hamed, N. A. Torquato, C. A. Tajon, B. Tian, B. Shevitski, E. S. Barnard, Y. D Suh, S. Aloni , J. B. Neaton, E. M. Chan, B. E. Cohen and P. J.Schuck, Nat. Photonics 12, 402-405 (2018).

26. Lian, Y. Fang, E. Joseph, Q. Wang, J. Li, S. Banerjee, C. Lollar, X. Wang and H.-C. Zhou, Chem. Soc. Rev. 46, 3386-3401 (2017).

27. Kitao, Y. Zhang, S. Kitagawa, B. Wang and T. Uemura, Chem. Soc. Rev. 46, 3108-3133 (2017).

28. Lu, T. Aung, N. Guo, R. Weichselbaum and W. Lin, Adv. Mater. 30, 1707634 (2018).

29. Carne, C. Carbonell, I. Imaz and D. Maspoch, Chem. Soc. Rev. 40, 291-305 (2011).

30 He X Dwan N Gun $C$. Chan $C$. Pnon R. Weichselbaum and W. Lin, Nat. Commun. 7, 12499 (2016). Loading [MathJax]/jax/output/CommonHTML/jax.js 
31. Ahmed and S. H. Jhung, Mater. Today 17, 136-146 (2014).

32. Zou, L. Wang, C. Zeng, X. Gao, Q. Wang and S. Zhong, Front. Mater. Sci. 12, 327-347 (2018).

33. Kumar, K. Vellingiri, K.-H. Kim, R. J. C. Brown and M. J. Manos, Micropor. Mesopor. Mat. 253, 251-265 (2017).

34. Jabbari, J. M. Veleta, M. Zarei-Chaleshton, J. Gardea-Torredey and D. Villagrán, Chem. Eng. J. 304, 774-783 (2016).

35. -L. Zhu and Q. Xu, Chem. Soc. Rev. 43, 5468-5512 (2014).

36. W. Li, X. J. Tian, Z. Q. Wang, Z. Guan, X. Q. Li, H. Qiao, H. Z. Ke, L. Luo and Q. F. Wei, Chem. Eng. J. 383, 123127 (2020).

37. Wang, C. Xu, L. Xu, C. Sun, D. Yang, J. Xu, F. He, S. Gai and P. Yang, J. Mater. Chem. B 6, 2597-2607 (2018).

38. Xu, D. Yang, W. Han, S. Dong, T. Jia, F. He, H. Bi, S. Gai, L. Li and P. Yang, J. Mater. Chem. C 6, 75337540 (2018).

39. Haase and H. Schäefer, Angew. Chem. Int. Ed. 50, 5808-5829 (2011).

40. Dai, Z. Zhong, X. Wu, S. Zhan, S. Hu, P. Hu, J. Hu, S. Wu, J. Han and Y. Liu, Nanotechnology 28, 155702 (2017).

41. Ding, C. H. Liow, M. Zhang, R. Huang, C. Li, H. Shen, M. Liu, Y. Zou, N. Gao, Z. Zhang, Y. Li, Q. Wang, S. Li and J. Jiang, J. Am. Chem. Soc. 136, 15684-15693 (2014).

42. Xu, H. Song, X. Chen, H. Wang, S. Cui, D. Zhou, P. Zhou and S. Xu, Chem. Commun. 51, 1502-1505 (2015).

43. Wang, Z. Yang, Y. Ma, Z. Chai, J. Qiu and Z. Song, J. Mater. Chem. C 5, 8535-8544 (2017).

44. Li, T. Meade, J. Am. Chem. Soc. 141, 17025-17041 (2019).

45. Perry, R. Botnar and J. Wilton-Ely, Chem. Commun. 56, 4037-4046 (2020).

46. Li, D. Yang, P. Ma, Y. Chen, Y. Wu, Z. Hou, Y. Dai, J. Zhao, C. Sui and J. Lin, Small 9, 4150-4159 (2013).

47. A. Agarwal and N. K. Gupta, RSC Adv. 7, 3870-3878 (2017).

48. Xu, K. Kattel, J. Y. Park, Y. Chang, T. J. Kim and G. H. Lee, Chem. Chem. Phys. 14, 12687-12700 (2012).

49. Li, P. Zhao and D. Astruc, Angew. Chem. Int. Ed. 53, 1756-1789 (2014).

\section{Figures}



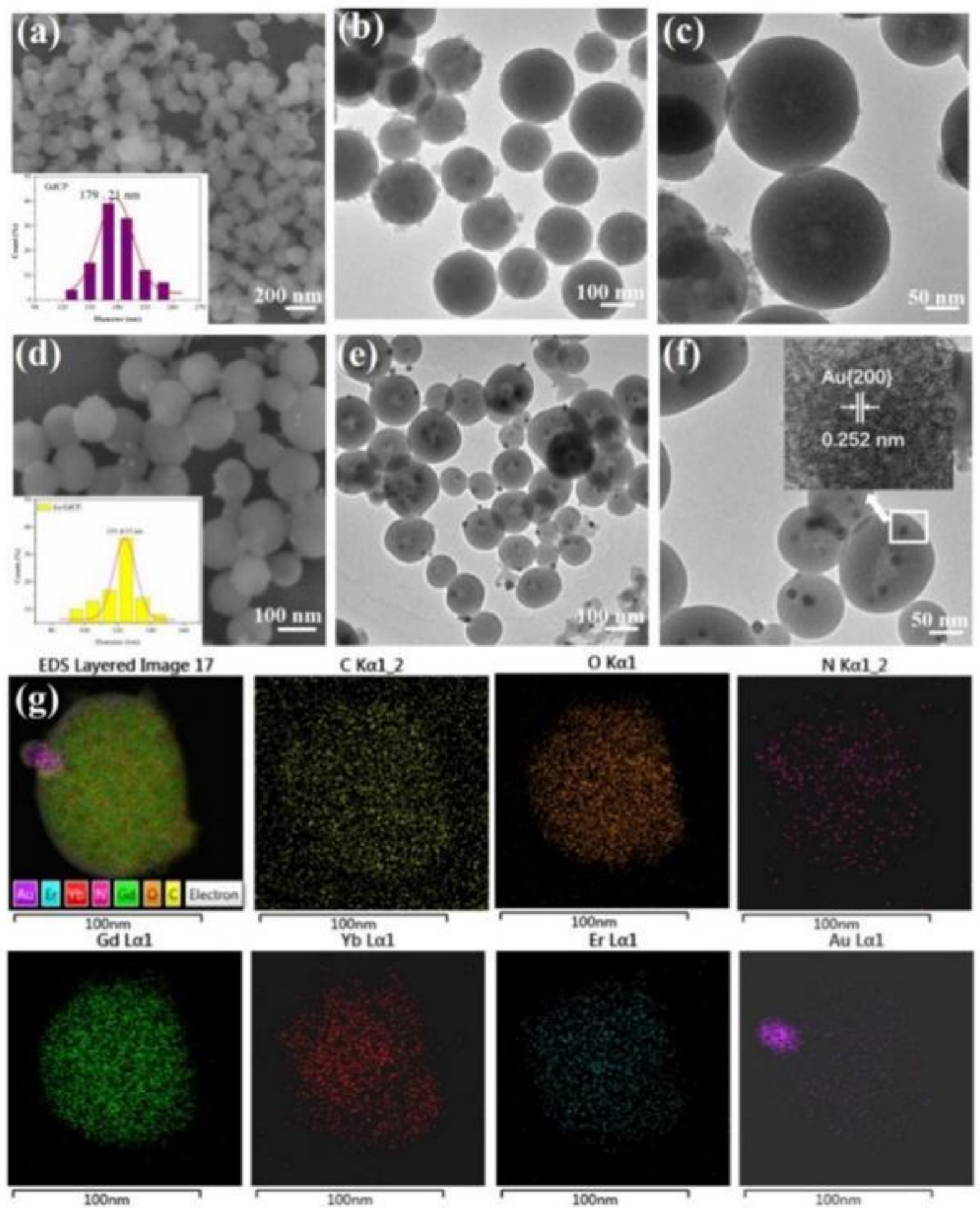

\section{Figure 1}

SEM (a) and TEM (b, c) images of GdCP. SEM (d) and TEM (e, f) images of Au@GdCP nanoparticles and energy dispersive spectroscopy (EDS) mapping spectra of Au@GdCP (g). 


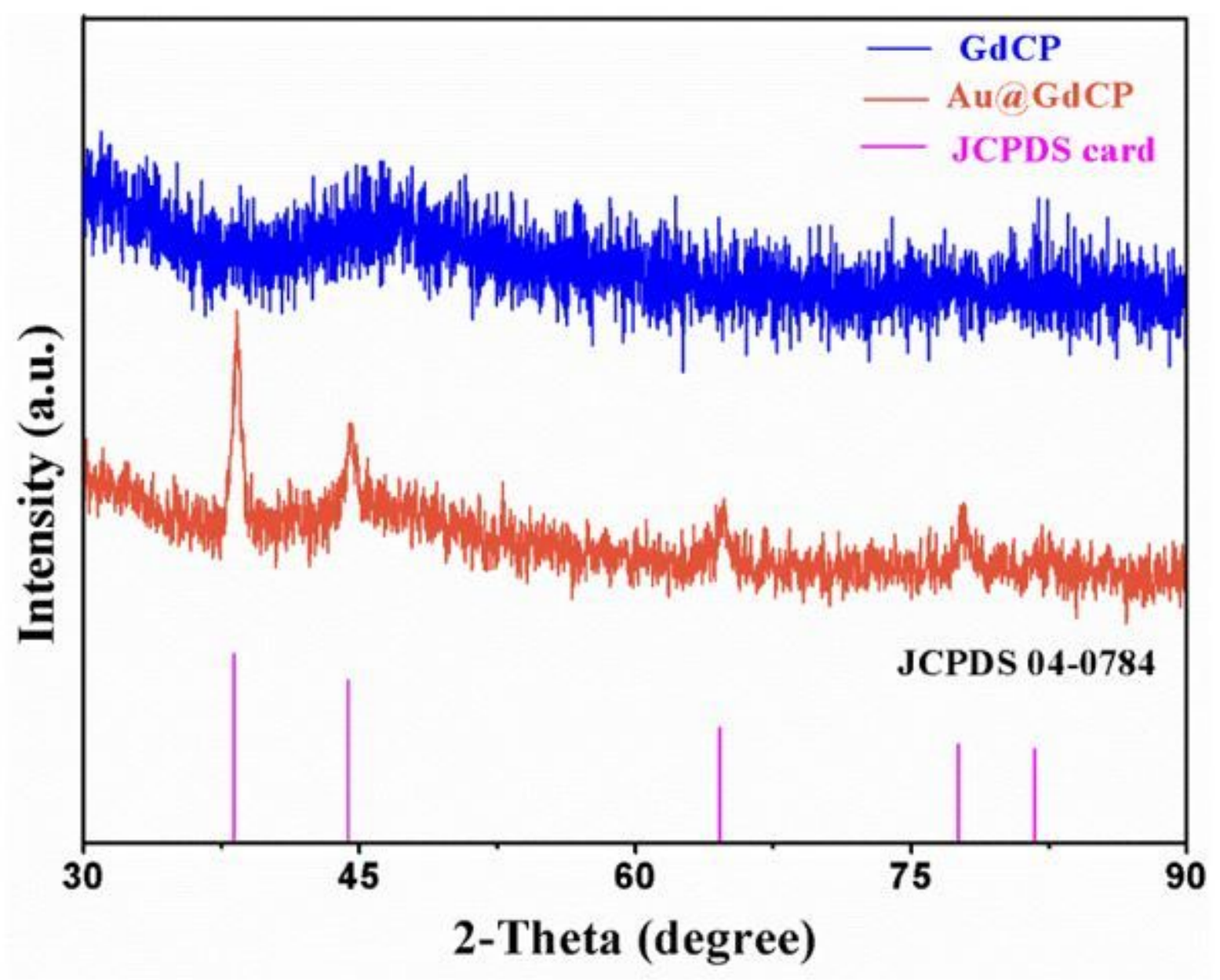

Figure 2

X-ray diffraction (XRD) patterns of GdCP and the Au@GdCP nanocomposite.
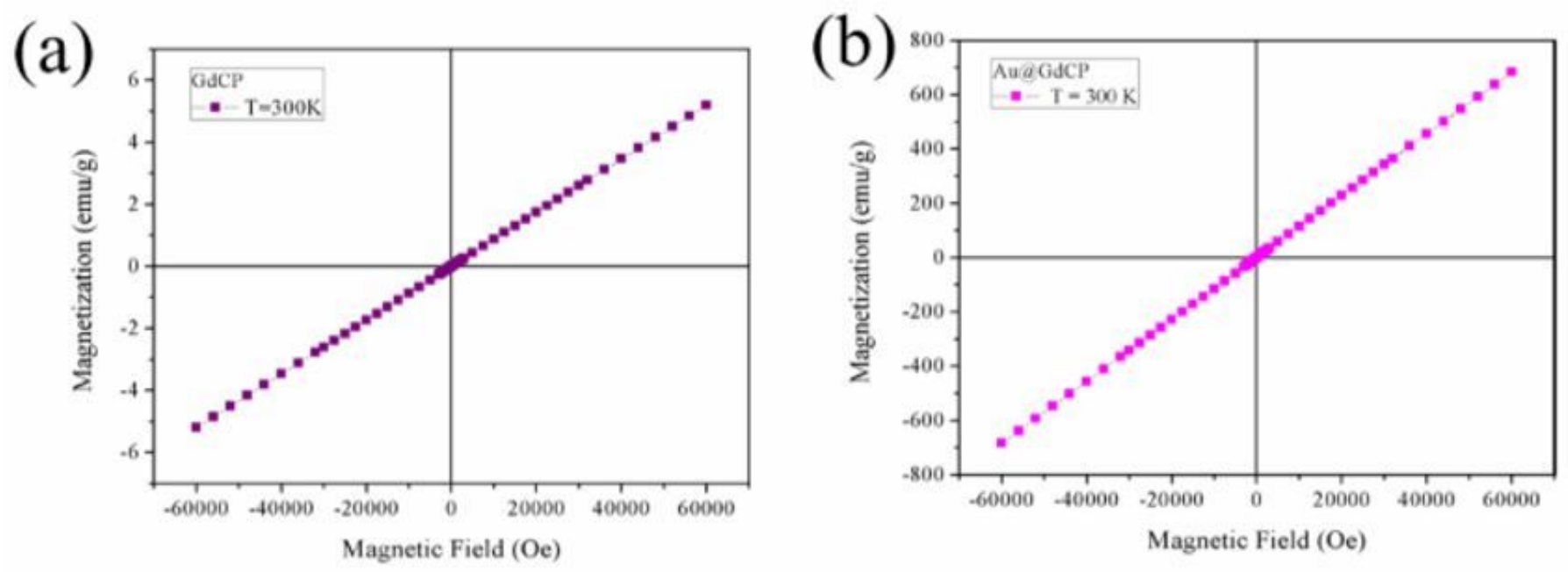

Figure 3

Loading [MathJax]/jax/output/CommonHTML/jax.js 
The magnetic properties of GdCP (a) and Au@GdCP(b).



Figure 4

Upconversion emission spectra for GdCP (a) and Au@GdCP (b). 
(a)

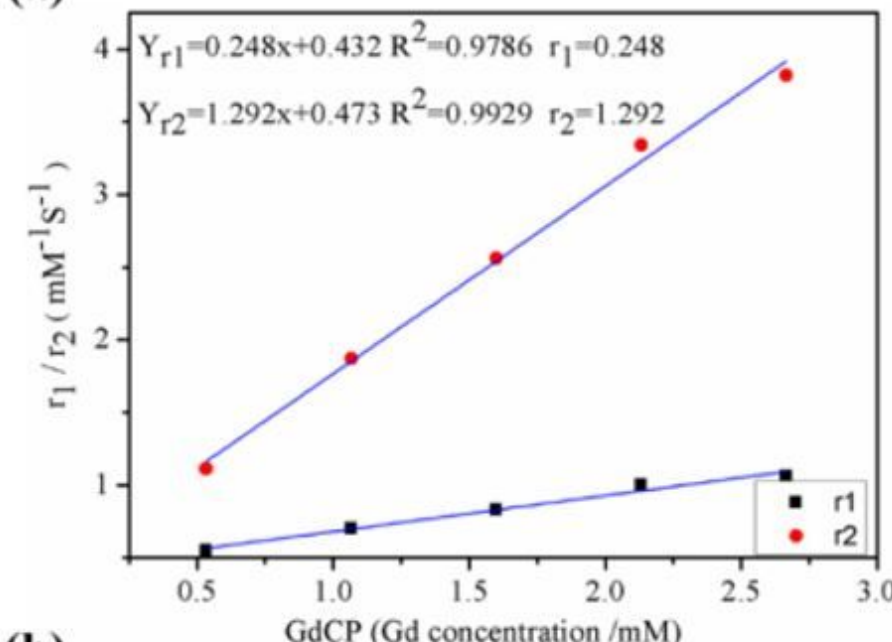

(b)

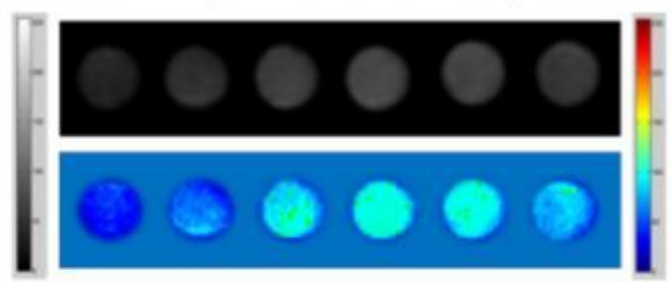

$0 \mathrm{mg} / \mathrm{ml}$ $1.0 \mathrm{~m} \mathrm{~g} / \mathrm{ml}$ (c)

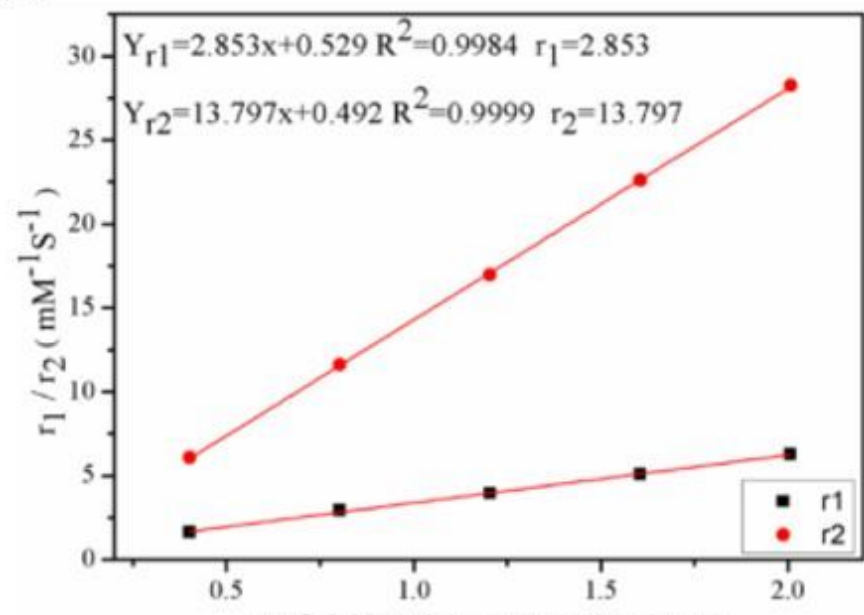

(d)

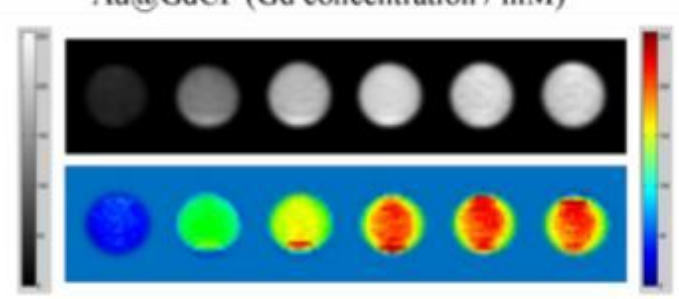

$0 \mathrm{mg} / \mathrm{ml}$

$1.0 \mathrm{mg} / \mathrm{ml}$

\section{Figure 5}

Relaxation information and T1-weighted MRI maps with different concentrations of $\operatorname{GdCP}(a, b)$ and $A u @ G d C P(c, d)$. 


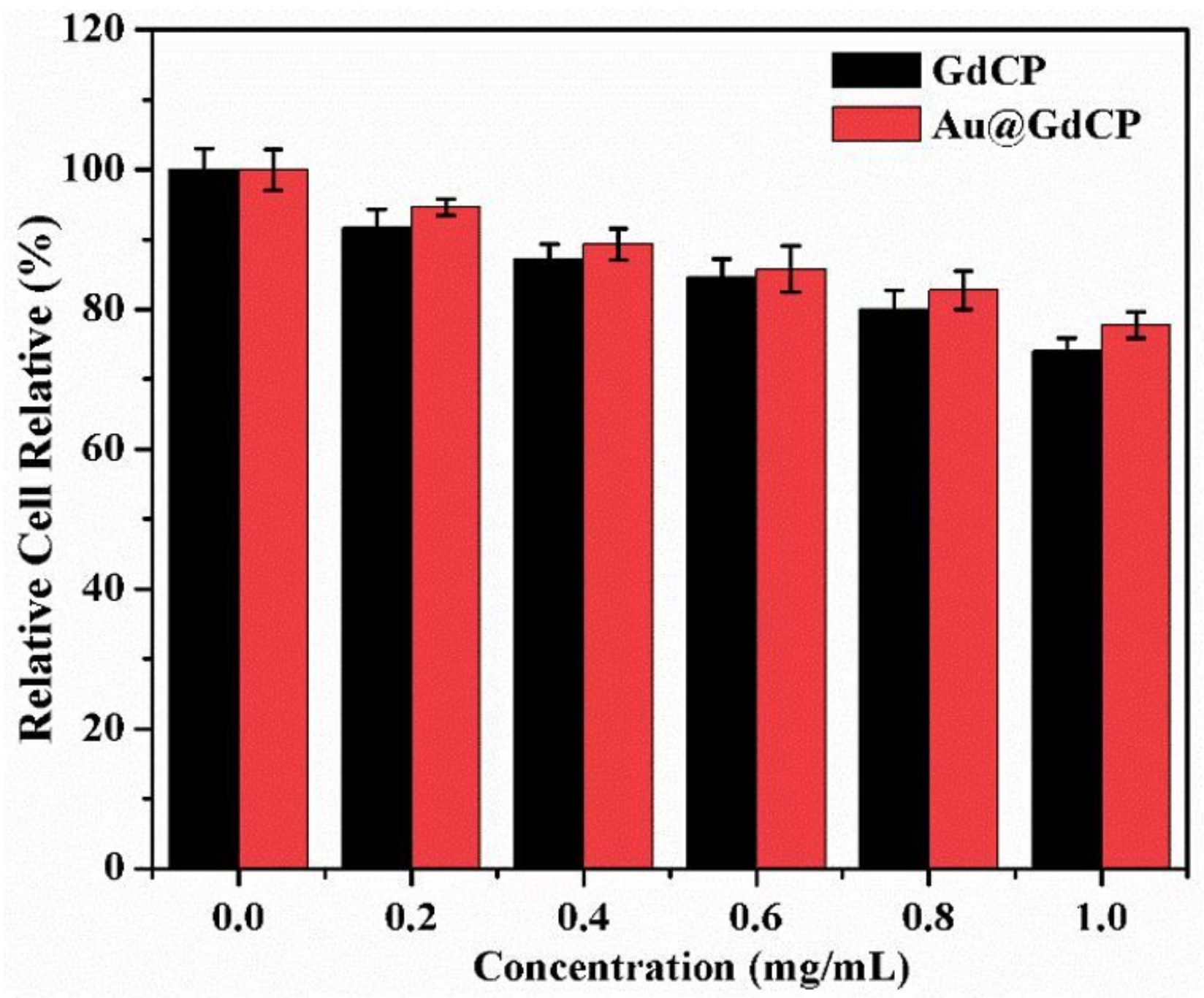

Figure 6

Relative cell viability of HeLa incubated with various concentrations of GdCP and Au@GdCP at $37^{\circ} \mathrm{C}$ for an incubation time of $24 \mathrm{~h}$. 